\title{
Asymptotic Analysis of the Local Potential Approximation to the Wetterich Equation
}

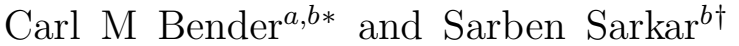 \\ ${ }^{a}$ Department of Physics, Washington University, St. Louis, Missouri 63130, USA \\ ${ }^{b}$ Department of Physics, King's College London, London WC2R 2LS, UK
}

\begin{abstract}
This paper reports a study of the nonlinear partial differential equation that arises in the local potential approximation to the Wetterich formulation of the functional renormalization group equation. A cut-off-dependent shift of the potential in this partial differential equation is performed. This shift allows a perturbative asymptotic treatment of the differential equation for large values of the infrared cut-off. To leading order in perturbation theory the differential equation becomes a heat equation, where the sign of the diffusion constant changes as the space-time dimension $D$ passes through 2. When $D<2$, one obtains a forward heat equation whose initial-value problem is well-posed. However, for $D>2$ one obtains a backward heat equation whose initial-value problem is ill-posed. For the special case $D=1$ the asymptotic series for cubic and quartic models is extrapolated to the small infrared-cut-off limit by using Padé techniques. The effective potential thus obtained from the partial differential equation is then used in a Schrödinger-equation setting to study the stability of the ground state. For cubic potentials it is found that this Padé procedure distinguishes between a $\mathcal{P} T$-symmetric $i g \phi^{3}$ theory and a conventional Hermitian $g \phi^{3}$ theory $(g$ real). For an $i g \phi^{3}$ theory the effective potential is nonsingular and has a stable ground state but for a conventional $g \phi^{3}$ theory the effective potential is singular. For a conventional Hermitian $g \phi^{4}$ theory and a $\mathcal{P} T$-symmetric $-g \phi^{4}$ theory $(g>0)$ the results are similar; the effective potentials in both cases are nonsingular and possess stable ground states.
\end{abstract}

*Electronic address: cmb@wustl.edu

${ }^{\dagger}$ Electronic address: sarben.sarkar@kcl.ac.uk 


\section{INTRODUCTION}

The renormalization group interpolates between microscopic and macroscopic scales. In the context of the renormalization group Wilson and Kadanoff pioneered a nonperturbative approach that led to a reformulation of earlier perturbative approaches to the role of scale and universality [1]. The Wilson-Kadanoff approach involves the construction of effective field theories in which short-distance fluctuations below a certain length scale are integrated out. Early work on effective actions that preserve universality may be found in Ref. [2].

A recent development, known as the functional renormalization group, combines the functional formulation of quantum field theory with the Wilsonian renormalization group [3] 5]. However, the functional-renormalization-group equations are intractable. This paper examines a version of the approach due to Wetterich [6], which in the potential approximation leads to a nonlinear partial differential equation (PDE) rather than a functional equation. This simplification enables substantial progress in understanding the effective potential in arbitrary space-time dimension.

Our purpose here is to examine the nonlinear PDE due to Wetterich. Our approach in Sec. II is unconventional and is based on an asymptotic analysis of the PDE for large values of the cut-off. This analysis allows us to make general observations regarding the wellposedness of the leading-order approximation to the PDE. For $D<2$ the approximation is well-posed but for $D>2$ the approximation is ill-posed. This observation is based on asymptotic analysis, but it does provide a clue to understanding the instabilities of numerical solutions for $D=4$ obtained by making polynomial truncations in effective potentials [7].

Next, we specialize to the quantum-mechanical case $D=1 \mathrm{in} \mathrm{Sec.} \mathrm{III} \mathrm{and} \mathrm{go} \mathrm{to} \mathrm{very}$ high order in our asymptotic approximation. We seek a solution to the Wetterich PDE as an asymptotic series in powers of the inverse momentum cut-off. We consider two cases: In the first case the leading term $g \phi^{3}$ is cubic in $\phi$. If $g$ is real, the potential is unbounded below and the energy levels are complex. However, if the leading term is $i g \phi^{3}$, where $g$ is real, this is a non-Hermitian but $\mathcal{P} T$-symmetric potential whose energy levels are real and bounded below [8, 9]. In the second case the leading term $g \phi^{4}$ is quartic in $\phi$. If $g>0$, the potential is bounded below and the energy spectrum is positive. If $g<0$, we interpret the potential as being $\mathcal{P} T$ symmetric, and again the energy levels are real and bounded below [8, 9]. The asymptotic expansion respects $\mathcal{P} T$ symmetry to all orders and allows us to 
investigate differences in the renormalization-group flow (for $D=1$ ) of the effective actions. We examine the stability of the effective potential in the limit as the infrared cut-off is extrapolated to zero by using Padé approximants. This procedure is similar in spirit to the high-temperature analysis of phase transitions in which Padé approximation is used to extrapolate from high temperature to finite temperature [10].

\section{Local potential approximation for the Wetterich functional equation}

For completeness we give a brief review of the derivation of the nonlinear PDE. The Euclidean action for scalar field theories, where the Fourier components of the field $\Phi$ have momenta of magnitude less than the ultraviolet cut-off $\Lambda$, is

$$
S_{\Lambda}[\Phi]=\int d^{D} x\left[\frac{1}{2} \partial_{\mu} \Phi \partial^{\mu} \Phi+V_{\Lambda}(\Phi)\right]
$$

where $D$ is the dimension of space-time. If we integrate over Fourier modes with momenta of magnitude $p$ in the shell $k \leq p \leq \Lambda$, we arrive at an action that we denote by $S_{k}[\phi]$, where $\phi$ has Fourier modes with $p \leq k$. Instead of a sharp infrared cut-off $k$, Wetterich introduced a smooth infrared cut-off function $R_{k}\left(p^{2}\right)$ [6], which leads to the concept of an average action $\mathcal{S}_{k}[\phi]$. We consider the renormalization group in the approximation that the average action $\mathcal{S}_{k}[\phi]$ is given by

$$
\mathcal{S}_{k}[\phi]=\int d^{D} x\left[\frac{1}{2} \partial_{\mu} \phi \partial^{\mu} \phi+V_{k}(\phi)\right]+\Delta_{k}[\phi],
$$

where $\Delta_{k}[\phi]=\frac{1}{2} \int d^{D} p \phi_{p} R_{k}\left(p^{2}\right) \phi_{-p}$.

A simple smooth cut-off function $R_{k}\left(p^{2}\right)=\left(k^{2}-p^{2}\right) \Theta\left(k^{2}-p^{2}\right)$ that embodies the properties required for $R_{k}\left(p^{2}\right)$ was introduced by Litim [11]. From the average partition function

$$
Z_{k}[j] \equiv \exp \left(-W_{k}[j]\right) \equiv \int \mathcal{D} \phi \exp \left(-\mathcal{S}_{k}[\phi]-\int_{p} j_{p} \phi_{-p}\right)
$$

the Legendre transformation gives the averaged effective action [5]

$$
\Gamma_{k}\left[\phi_{c}\right]=W_{k}[j]-\Delta_{k}\left[\phi_{c}\right]-\int d^{D} x j \phi_{c}
$$

The renormalization group flow equation is [5]

$$
\partial_{k} \Gamma_{k}\left[\phi_{c}\right]=\frac{1}{2} \operatorname{Tr}\left\{\partial_{k} R_{k}\left(\frac{\delta^{2} \Gamma_{k}}{\delta \phi_{c}(p) \delta \phi_{c}(q)}+R_{k}\left(p^{2}\right) \delta(p+q)\right)^{-1}\right\}
$$

To obtain the Wetterich equation we then make the potential approximation ansatz

$$
\Gamma_{k}\left[\phi_{c}\right]=\int d^{D} x\left(\frac{1}{2} \partial_{\mu} \phi_{c} \partial^{\mu} \phi_{c}+U_{k}\left(\phi_{c}\right)\right)
$$


for the average effective action with Litim's form for $R_{k}(p)$. The flow equation then becomes

$$
\partial_{k} U_{k}\left(\phi_{c}\right)=\frac{1}{\pi_{D}} \frac{k^{D+1}}{k^{2}+U_{k}^{\prime \prime}\left(\phi_{c}\right)},
$$

where $U_{k}^{\prime \prime}\left(\phi_{c}\right) \equiv \frac{d^{2}}{d \phi_{c}^{2}} U_{k}\left(\phi_{c}\right), \pi_{D}=\frac{D(2 \pi)^{D}}{S_{D-1}}$, and $S_{D-1}=\frac{2 \pi^{D / 2}}{\Gamma(D / 2)}$ is the surface area of a unit $D$-dimensional sphere. This is the $D$-dimensional form of the PDE examined in this paper.

We may assume that the equations for $U_{k}\left(\phi_{c}\right)$ involve dimensionless quantities. (If this were not so, we could introduce a mass scale $M$ to achieve dimensionless variables. For example, for $D=1$ the dimensionless variables, denoted by a tilde, are $\tilde{\phi}=M^{1 / 2} \phi, \tilde{g}=$ $M^{-3} g, \tilde{k}=M^{-1} k$, and $\tilde{\mu}=M^{-1} \mu$.) The Wetterich equation (7) can be viewed as being in terms of such dimensionless variables.

To avoid numerical difficulties associated with boundary conditions, in the past the PDE (7) has been analyzed by approximating $U_{k}\left(\phi_{c}\right)$ as a finite series in powers of the field $\phi_{c}$. This ansatz leads to a sequence of coupled nonlinear ordinary differential equations (see, for example, Ref. 4]). The consistency of such a procedure has not been rigorously established.

For the quantum-mechanical case $(D=1)(7)$ becomes

$$
\partial_{k} U_{k}\left(\phi_{c}\right)=\frac{1}{32 \pi^{2}} \frac{k^{2}}{k^{2}+U_{k}^{\prime \prime}\left(\phi_{c}\right)} .
$$

Even in this one-dimensional setting, no exact solution to this nonlinear PDE is known and only numerical solutions have been discussed [4].

\section{LARGE CUT-OFF ANALYSIS OF THE WETTERICH EQUATION}

In this paper we depart from the usual treatment of the Wetterich potential equation (7) by performing an asymptotic analysis for large values of the cut-off $k$. This avoids the arbitrariness involved in restricting the trajectory of the potential to a truncated function space required in the usual treatment [5]. Consequently, our approach also avoids the appearance of coupled nonlinear ordinary differential equations [4]. To leading order the results of this analysis are qualitatively different depending on whether the space-time dimension $D$ is greater than or less than 2 .

Letting $z=k^{2+D}$ and $U_{k}\left(\phi_{c}\right)=U(z, \phi)$, we can rewrite (7) as

$$
U_{z}(z, \phi)=\frac{1}{(2+D) \pi_{D}} \frac{1}{z^{\frac{2}{2+D}}+U_{\phi \phi}(z, \phi)},
$$


where the subscripts on $U$ indicate partial derivatives. We assume that for large $z$ we may neglect the $U_{\phi \phi}$ term in the denominator. (The consistency of this assumption is easy to verify if $D<2$.) To leading order in our approximation scheme we get

$$
U_{z}(z, \phi) \sim \frac{1}{(2+D) \pi_{D}} z^{-\frac{2}{2+D}} \quad(z \gg 1)
$$

On incorporating a correction $\epsilon$ to this leading behavior

$$
U(z, \phi)=\frac{1}{D \pi_{D}} z^{\frac{D}{2+D}}+\epsilon(z, \phi)
$$

we get to order $O\left(\epsilon^{2}\right)$

$$
\epsilon_{z}(z, \phi)=-\frac{1}{(D+2) \pi_{D}} z^{-\frac{4}{2+D}} \epsilon_{\phi \phi}(z, \phi) .
$$

On making the further change of variable

$$
t=\frac{D+2}{2-D} z^{\frac{D-2}{D+2}}
$$

(12) becomes

$$
\epsilon_{t}(t, \phi)=\frac{1}{(D+2) \pi_{D}} \epsilon_{\phi \phi}(t, \phi) .
$$

The variable $t$ in (13) is positive for $D<2$ and negative for $D>2$ and is not defined at $D=2$. Thus, (14) is a conventional diffusion equation for $D<2$ but is a backward diffusion equation for $D>2$. The backward diffusion equation is an inverse problem that is ill-posed [12. The problems associated with this ill-posedness may be connected with difficulties in solving (7) numerically when $D=4$ [3, 7].

If $D<2$ in (14), $t$ is positive and $t \rightarrow \infty$ as $z \rightarrow 0$. This includes the special case $D=1$, which is investigated in Sec. III. However, for $D>2$ we can set $\tau=-t$ so that $\tau \rightarrow \infty$ as $z \rightarrow \infty$. In terms of the $\tau$ variable we obtain the backwards diffusion equation

$$
\partial_{\tau} \epsilon=-\frac{1}{(D+2) \pi_{D}} \epsilon_{\phi \phi} .
$$

A well-posed differential-equation problem, as discussed by Hadamard, has three characteristics: (i) The solution exists; (ii) the solution is unique; (iii) the solution is stable with respect to small variations of the initial data. If these three conditions are not met, the differential-equation problem is ill-posed.

The $\phi$ variable in the heat diffusion equation (14) can be rescaled so that the equation appears in the standard form: $\epsilon_{t}=\epsilon_{\phi \phi}$. To demonstrate the stability of the forward diffusion 
equation $u_{t}=u_{x x}$ and the instability of the backward diffusion equation $u_{t}=-u_{x x}$ we need only consider a small perturbation of the initial data $u(x, 0) \rightarrow u(x, 0)+\Delta u(x, 0)$, which gives rise to a change in the solution at time $t$ :

$$
u(x, t) \rightarrow u(x, t)+\Delta u(x, t)
$$

For simplicity, we take $\Delta u(x, t)=\delta e^{a t} e^{i b x}$, where for $\delta \ll 1$ this is a small perturbation of the initial data. Then the forward heat equation gives the dispersion relation $a=-b^{2}$, so

$$
\Delta u(x, t)=\delta e^{-b^{2} t} e^{i b x}
$$

Since the heat equation is linear we can use a Fourier representation to construct any small perturbation of the initial data as a linear combination of terms of the form $e^{i b x}$. We can see from (16) that perturbing the initial data by an amount proportional to $\delta$ gives a perturbation of the solution for $t>0$ of order $\delta$. Thus, a small change in the initial data produces a small change in the solution at later times.

The backward heat equation gives the dispersion relation $a=b^{2} \mathrm{R}$, so a small change in the initial data of the form $u(x, 0) \rightarrow u(x, 0)+\delta e^{i b x}$ gives rise to a solution that at later times grows exponentially with $t$ like $e^{b^{2} t}$. So, small changes in the initial data produce arbitrarily large changes in the solution and the backwards heat-equation problem is ill-posed.

\section{SERIES EXPANSIONS AND EXTRAPOLATION FOR $D=1$}

The discussion in Sec. II shows that instability problems arise when $D>2$. These problems may just be an artifact of the nonperturbative expansion techniques proposed in this paper, or perhaps they have a more fundamental origin. However, there is no difficulty in applying our method in low-dimensional quantum field theory. In this section we apply our techniques to the quantum-mechanical case $D=1$. We are especially interested to see what happens if they are applied to $\mathcal{P} T$-symmetric quantum-mechanical theories.

On defining $\widehat{U}_{k}\left(\phi_{c}\right) \equiv U_{k}\left(\phi_{c}\right)-\frac{k}{\pi}, 8$ becomes

$$
\partial_{k} \widehat{U}_{k}\left(\phi_{c}\right)=-\frac{\widehat{U}_{k}^{\prime \prime}\left(\phi_{c}\right)}{\pi\left[k^{2}+\widehat{U}_{k}^{\prime \prime}\left(\phi_{c}\right)\right]} .
$$

From 17] it is consistent to assume that $\widehat{U}\left(\phi_{c}\right) \rightarrow V\left(\phi_{c}\right)$ and $\widehat{U}^{\prime \prime}\left(\phi_{c}\right) / k^{2} \rightarrow 0$ as $k \rightarrow \infty$. (For notational simplicity we have dropped the suffix $k$ on $\widehat{U}_{k}$.) The correction to this asymptotic 
behavior is $V\left(\phi_{c}\right)+\frac{1}{k} U_{1}\left(\phi_{c}\right)$ and substituting in 17$)$ gives

$$
U_{1}\left(\phi_{c}\right)=\frac{1}{\pi} V^{\prime \prime}\left(\phi_{c}\right)
$$

The next corrections are $\widehat{U}\left(\phi_{c}\right)=V\left(\phi_{c}\right)+\frac{1}{k \pi} V^{\prime \prime}\left(\phi_{c}\right)+\frac{1}{k^{2}} U_{2}\left(\phi_{c}\right)+\frac{1}{k^{3}} U_{3}\left(\phi_{c}\right)$ and we get

$$
U_{2}\left(\phi_{c}\right)=\frac{1}{2 \pi^{2}} V^{(4)}\left(\phi_{c}\right) \quad \text { and } \quad U_{3}\left(\phi_{c}\right)=\frac{1}{6 \pi^{3}} V^{(6)}\left(\phi_{c}\right)-\frac{1}{3}\left[V^{(2)}\left(\phi_{c}\right)\right]^{2},
$$

where $V^{(n)}$ is the $n$th derivative of $V$. To formalize this procedure we let $\delta=\pi / k$ and $\phi=\pi \phi_{c}$ so that 17 becomes

$$
\frac{\partial}{\partial \delta} \widehat{U}=\frac{\frac{\partial^{2}}{\partial \phi^{2}} \widehat{U}}{1+\delta^{2} \frac{\partial^{2}}{\partial \phi^{2}} \widehat{U}}, \text { where } \widehat{U}=\sum_{n=0}^{\infty} \delta^{n} U_{n}(\phi) .
$$

[A similar analysis of (17) can be performed for $D=2-\eta$ with $\eta$ positive and small: $\widehat{U}(\phi) \sim \sum_{n} a_{n}(\phi) k^{-n \eta}$. This case will be studied elsewhere.]

Our perturbation expansion in powers of $\delta$, where $\delta \ll 1$ and $k \gg 1$, probes the effective potential at microscopic scales. However, we want to probe the infrared limit $\delta \rightarrow \infty$ of the

effective potential. The generic problem here is obtaining the large- $\delta$ behavior of $\hat{U}$ from a formal power series that is valid for small $\delta$. There is a parallel in the theory of phase transitions; there, a physical entity is expanded in inverse powers of the temperature $T$ [18] and an extrapolation procedure is used to find the critical behavior at small $T$.

A powerful extrapolation technique used here to deduce the large- $\delta$ behavior from the small- $\delta$ behavior makes use of Padé approximation [19, 20]. Padé approximation consists of converting the formal power series $\sum_{n} a_{n} \delta^{n}$ to a sequence of rational functions

$$
P_{M}^{N}(\delta)=\frac{\sum_{n=0}^{N} A_{n} \delta^{n}}{\sum_{n=0}^{M} B_{n} \delta^{n}} .
$$

The advantage of constructing $P_{M}^{N}(\delta)$ is that in many instances $P_{M}^{N}(\delta)$ is a convergent sequence as $N, M \rightarrow \infty$ even when $\sum_{n} a_{n} \delta^{n}$ is a divergent series.

\section{A. Nonperturbative extrapolation of the series expansion for $D=1$}

Our treatment thus far has been for a general potential. However, we now specialize to two cases, $U_{0}(\phi)=g \phi^{3}$, which is $\mathcal{P} T$ symmetric for $g=i$, and $U_{0}(\phi)=g \phi^{4}$, which $\mathcal{P} T$ symmetric for $g$ real and negative [8, 16, 17]. We also study the effect of including a mass term of the form $\mu^{2} \phi^{2}$ in $U_{0}(\phi)$ for both the cubic and quartic cases. 
$\mathcal{P} T$-symmetric field theories are invariant under combined space and time reflection. The symbol $\mathcal{P}$ indicates space reflection (parity) and the symbol $\mathcal{T}$ indicates time reversal. $\mathcal{P} T$-symmetric field theories are complex non-Hermitian deformations of conventional Hermitian field theories in which the deformation preserves the reality of the eigenvalues. The advantage of a spectral-reality-preserving deformation is that a new inner product on the Hilbert space of states can be constructed with respect to which the time evolution is unitary [8]. A remarkable feature of $\mathcal{P} T$-symmetric theories is that while they may appear to be unstable, the energy spectrum is real and bounded below, and thus there is actually no instability [8]. It is interesting that when one renormalizes a quantum field theory by inserting counterterms, the effect of doing so can induce non-Hermiticity in the Hamiltonian for the field theory even though the unrenormalized Hamiltonian is formally Hermitian. In some cases, when this happens, the appropriate approach is to reinterpret the resulting non-Hermitian Hamiltonian as being $\mathcal{P} T$ symmetric [13 17]. In this paper the nonperturbative functional-renormalization-group equation in the potential approximation is applied to some elementary $\mathcal{P} T$-symmetric field theory models for the first time. This nonperturbative treatment complements our earlier perturbative study of vacuum instabilities manifest in one-loop generated effective potentials [16].

\section{B. Cubic potentials}

We consider both massless and massive cubic potentials. In the former case $U_{0}(\phi)=g \phi^{3}$ and in the latter case $U_{0}(\phi)=\mu^{2} \phi^{2}+g \phi^{3}$. For the massless case the solution to (19) is

$$
\begin{aligned}
U_{1}(\phi) & =6 g \phi, & U_{10}(\phi) & =\frac{1621728}{175} g^{5} \phi^{3}, \\
U_{2}(\phi) & =0, & U_{11}(\phi) & =\frac{17882208}{1925} g^{5} \phi-\frac{46656}{11} g^{6} \phi^{6}, \\
U_{3}(\phi) & =-12 g^{2} \phi^{2}, & U_{12}(\phi) & =-\frac{30608064}{385} g^{6} \phi^{4}, \\
U_{4}(\phi) & =-6 g^{2}, & U_{13}(\phi) & =\frac{279936}{13} g^{7} \phi^{7}-\frac{4537967328}{25025} g^{6} \phi^{2}, \\
U_{5}(\phi) & =\frac{216}{5} g^{3} \phi^{3}, & U_{14}(\phi) & =\frac{4512754944}{7007} g^{7} \phi^{5}-\frac{5900745888}{175175} g^{6}, \\
U_{6}(\phi) & =\frac{456}{5} g^{3} \phi, & U_{15}(\phi) & =\frac{1439488599552}{525525} g^{7} \phi^{3}-\frac{1679616}{15} g^{8} \phi^{8}, \\
U_{7}(\phi) & =-\frac{1296}{7} g^{4} \phi^{4}, & U_{16}(\phi) & =\frac{295324339824}{175175} g^{7} \phi-\frac{25063743168}{5005} g^{8} \phi^{6}, \\
U_{8}(\phi) & =-\frac{34668}{35} g^{4} \phi^{2}, & U_{17}(\phi) & =\frac{10077696}{17} g^{9} \phi^{9}-\frac{105103706989824}{2977975} g^{8} \phi^{4}, \\
U_{9}(\phi) & =\frac{7776}{9} g^{5} \phi^{5}-\frac{89496}{315} g^{4}, & U_{18}(\phi) & =\frac{3212310887424}{85085} g^{9} \phi^{7}-\frac{101014620538752}{2127125} g^{8} \phi^{2} .
\end{aligned}
$$

These expressions, which are valid for pure imaginary $g$ as well as for real $g$, are cumber- 
some but manageable. We stress that there has been no truncation of the function space on which $\widehat{U}(\phi)$ has support. This contrasts with the usual approach (for example, see Ref. [4]), which requires a truncation at the onset of the calculation of the renormalization group flow. This absence of truncation continues to be a feature for the massive case.

The iterative solution to (19) for the massive case is similar to that for the massless case, but the expressions for $U_{n}(\phi)$ have many more terms. For example, the coefficient of $\delta^{9}$ is

$$
\begin{gathered}
U_{9}(\phi)=\frac{8}{315}\left(34020 g^{5} \phi^{5}+56700 g^{4} \mu^{2} \phi^{4}-11187 g^{4}+37800 g^{3} \mu^{4} \phi^{3}\right. \\
\left.+12600 g^{2} \mu^{6} \phi^{2}+2100 g \mu^{8} \phi+140 \mu^{10}\right),
\end{gathered}
$$

which in the massless limit $\mu \rightarrow 0$ reduces to the two-term expression in 21). We refrain from listing the coefficients explicitly and instead proceed directly to the large- $\delta$ behavior of the diagonal Padé approximants. We denote the diagonal Padé approximants in the massless case by $P_{N}^{0, N}(\delta)$ and in the massive case by $P_{N}^{N}(\delta)$. (This nonperturbative analysis complements the perturbative analysis given in Refs. [16, 17].)

Let us examine some low-order Padé approximants in the limit $\delta \rightarrow \infty$. For example,

$$
\lim _{\delta \rightarrow \infty} P_{2}^{2}(\delta)=\frac{18 g^{3} \phi^{5}+3 g^{2}\left(10 \mu^{2} \phi^{4}-9\right)+14 g \mu^{4} \phi^{3}+2 \mu^{6} \phi^{2}}{2\left(3 g \phi+\mu^{2}\right)^{2}} .
$$

[As a check, when $\mu=0$ this expression agrees with the corresponding expression $g \phi^{3}-\frac{3}{2 \phi^{2}}$ for $P_{2}^{0,2}(\delta)$.] For $N=3$ we obtain

$$
\lim _{\delta \rightarrow \infty} P_{3}^{0,3}(\phi)=\frac{g \phi^{3}\left(736 g \phi^{5}+1705\right)}{25-800 g \phi^{5}} \quad \text { and } \quad P_{3}^{3}(\phi)=-u_{3} / d_{3},
$$

where

$$
\begin{gathered}
u_{3}=1609632 g^{8} \phi^{8}+729 g^{7} \phi^{3}\left(5888 \mu^{2} \phi^{4}+5115\right)+243 g^{6} \mu^{2} \phi^{2}\left(23008 \mu^{2} \phi^{4}+15345\right) \\
+1296 g^{5} \mu^{4} \phi\left(3376 \mu^{2} \phi^{4}+945\right)+15120 g^{4} \mu^{6}\left(142 \mu^{2} \phi^{4}+9\right)+658944 g^{3} \mu^{10} \phi^{3} \\
+121824 g^{2} \mu^{12} \phi^{2}+12288 g \mu^{14} \phi+512 \mu^{16}, \\
d_{3}=225 g^{2}\left[7776 g^{5} \phi^{5}+81 g^{4}\left(160 \mu^{2} \phi^{4}-3\right)+8640 g^{3} \mu^{4} \phi^{3}+2880 g^{2} \mu^{6} \phi^{2}+480 g \mu^{8} \phi+32 \mu^{10}\right] .
\end{gathered}
$$

[As a check, we have $\lim _{\mu \rightarrow 0} P_{3}^{3}(\phi)=P_{3}^{0,3}(\phi)$.]

Note that for the massless case $P_{2}^{0,2}$ has a double pole for both real and imaginary $g$. In fact, the same is true for $P_{n}^{0, n}$ for even $n$. This pole is an artifact of the Padé approximation and is not present when $n$ is odd, so we will only consider the behavior of these approximants 
for odd $n$. In general, the odd- $n$ diagonal Padé approximants have no singularities at all on the real- $\phi$ axis when $g$ is imaginary (see Fig 1 for the case $n=5$ ) but singularities occur for the case of real $g$ (see Fig. 2). These findings indicate that the $\mathcal{P} T$-symmetric effective potential is well behaved in the infrared limit (see Fig. 1). From the expressions for the diagonal Padé approximants we see that for large $|\phi|$, the leading behavior of the imaginary part of the effective potential is exactly $i \phi^{3}$. Consequently the $\mathcal{P} T$ nature of the interaction is preserved under renormalization (see the discussion of the effective potential obtained from the perturbative renormalization group in [16, 17]).
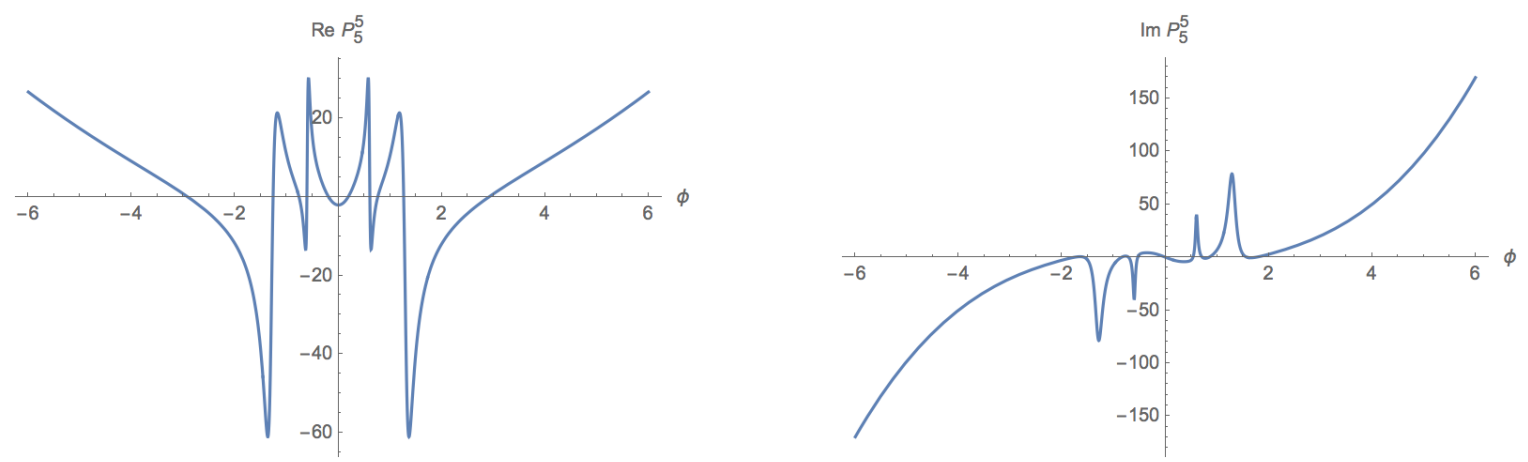

FIG. 1: Effective potential flow for massive $i \phi^{3}$ with the mass parameter $\mu=1$. Shown is a plot of the real and imaginary parts of the $P_{5}^{5}$ approximant plotted as functions of real $\phi$. Observe that there are no poles. The real part of the potential is right-side-up, so there is no instability. Furthermore, apart from small fluctuations near the origin, the imaginary part of the potential behaves like $i \phi^{3}$ for large $|\phi|$.

The situation is not so nice for the conventional $g \phi^{3}$ theory $(g$ real). In Fig. 2 we plot the Padé approximations $P_{5}^{5}$ for the effective potential flow for massive $\phi^{3}$ and $P_{7}^{7}$ for massless $\phi^{3}$. These plots indicate that poles appear on the real- $\phi$ axis. We emphasize that there are no such poles for the $\mathcal{P} T$-symmetric $i \phi^{3}$ theory. Furthermore, these potentials rise for positive $\phi$ and fall for negative $\phi$, which indicates that the theory is unstable. 

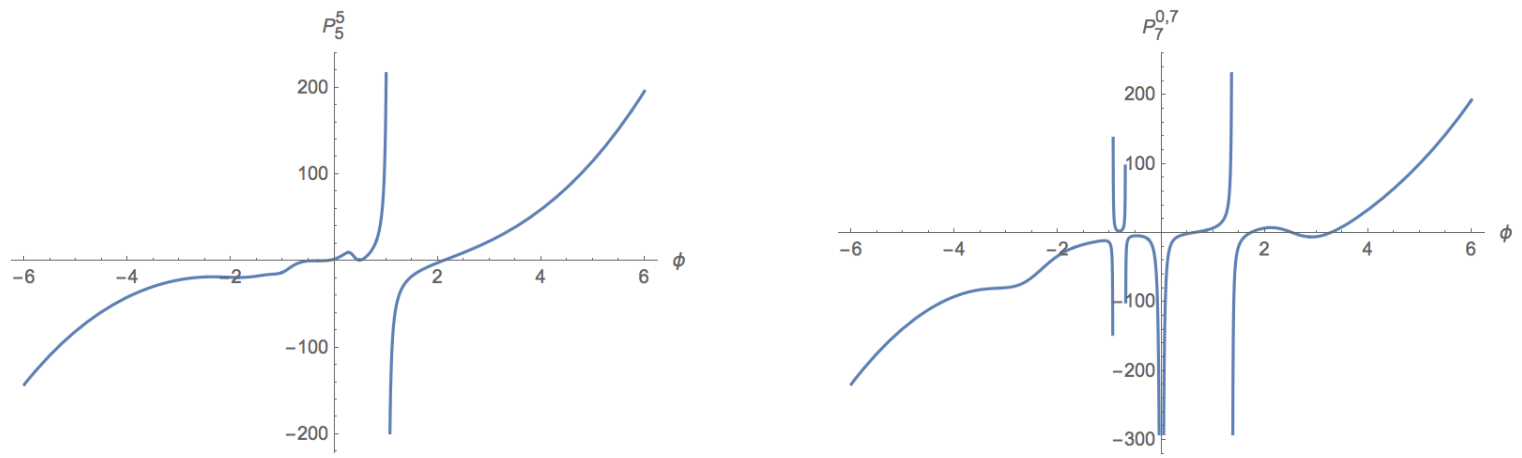

FIG. 2: Padé $P_{5}^{5}$ approximation for the effective potential flow for massive $\phi^{3}$ and $P_{7}^{7}$ for massless $\phi^{3}$ potentials. These potentials rise on the right but fall on the left. Thus, this theory is unstable and nonconfining. Note also that there are poles on the $\phi$ axis. There are no such poles for the $\mathcal{P} T$-symmetric $i \phi^{3}$ theory, as we can see in Fig. 1.

\section{Padé approximants for $\mathcal{P} T$-symmetric quartic theories}

We consider next the case of massless and massive $\mathcal{P} T$-symmetric quartic theories; that is, theories with potentials of the form $-g \phi^{4}$ and $\mu^{2} \phi^{2}-g \phi^{4}$. Potentials such as these are obtained from the general one-parameter class of potentials of the form $\phi^{2}(i \phi)^{\epsilon}$ and $\mu^{2} \phi^{2}+\phi^{2}(i \phi)^{\varepsilon}$, where $\varepsilon \geq 0$. These potentials interpolate between the quadratic and the cubic and quartic $\mathcal{P} T$-symmetric potentials considered in this paper: For $\varepsilon=2$ we obtain the negative-quartic potential and for $\varepsilon=1$ we obtain the imaginary cubic potential [8]. The functional integral representing the partition function for a $\mathcal{P} T$-symmetric $-\phi^{4}$ theory must be defined inside a pair of Stokes sectors of opening angle $\frac{\pi}{4}$ that are centered about the angles $\frac{\pi}{4}$ below the positive-real- $\phi$ axis and the negative-real- $\phi$.

To be precise, as soon as $\varepsilon>0$, a logarithmic branch point appears at the origin $\phi=0$. We choose the branch cut to run up the imaginary- $\phi$ axis from $\phi=0$ to $\phi=i \infty$. In this cut plane the integrand of the functional integral is single valued. The Stokes sectors in which the functional integral converges rotate downward into the complex- $\phi$ plane and the opening angles of the sectors decrease as $\varepsilon$ increases. (There are, in fact, many sectors inside of which the functional integral converges, but we choose to continue the field theory off the conventional free field theory (the harmonic oscillator for the case $D=1$ ), which is obtained 
when we set $\varepsilon=0$. For $\varepsilon>0$ the center lines of the left and right sectors lie at the angles

$$
\theta_{\text {left }}=-\pi+\frac{\pi \varepsilon}{2 \varepsilon+4} \quad \text { and } \quad \theta_{\text {right }}=-\frac{\pi \varepsilon}{2 \varepsilon+4} .
$$

The opening angle of each sector is $\Delta=\pi /(2+\varepsilon)$.

The functional integral may be performed along any contour in the complex- $\phi$ plane so long as the endpoints of the contour lie inside the left and right sectors. When $0 \leq \varepsilon<1$ the sectors contain the real axis. However, if $\varepsilon \geq 1$, the sectors rotate below the real- $\phi$ axis. In Fig. 3 we plot the massive quartic potential $-\phi^{4}+\phi^{2}$ along the center line in the right Stokes sector. Note that the real part of the potential rises along this; this is why the functional integral for the partition function converges for the upside-down quartic potential.
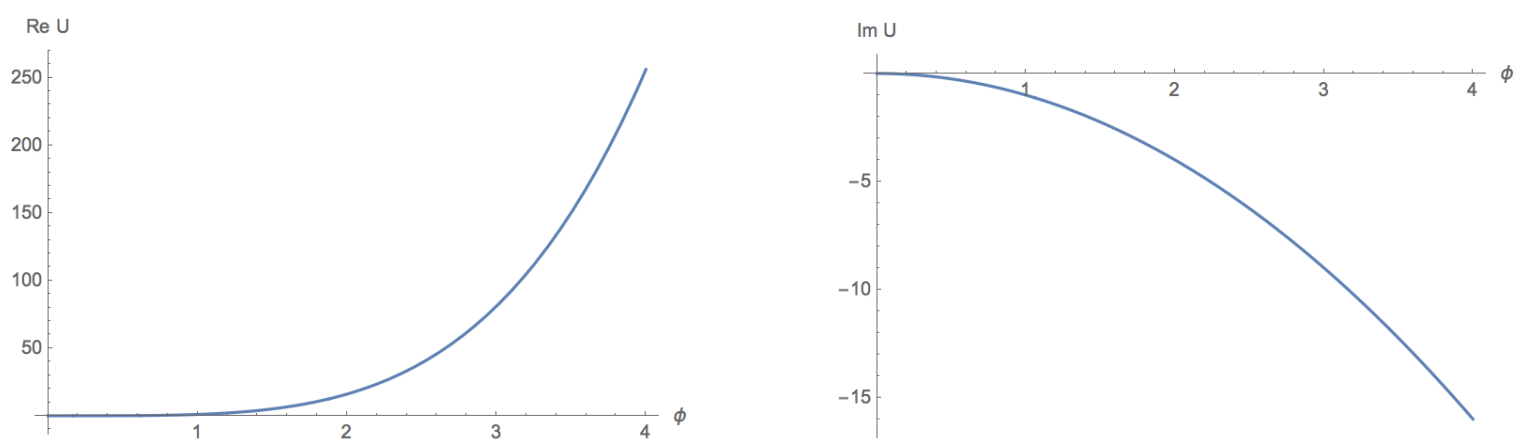

FIG. 3: Real and imaginary parts of the quartic potential $-\phi^{4}+\phi^{2}$ along the center line of the right Stokes sector. The functional integral converges because the real part of the potential rises along this line.

When we compute the Padé approximants for the $\mathcal{P} T$-symmetric (negative-quarticpotential) case, we find that there are poles at the origin for $P_{2}^{2}, P_{5}^{5}, P_{8}^{8}$, and so on. We interpret this problem as an artifact of the Padé procedure, and we disregard every third Padé approximant. (We observe an interesting pattern here: Every second Padé must be discarded for the cubic potential and every third Padé must be disregarded for the quartic potential.) The large- $|\phi|$ behavior of the Padé approximants has the form $\alpha g \phi^{4}$. From $P_{3}^{3}(\phi)$ we find that $\alpha=33 / 97=0.340206 \ldots$; from $P_{4}^{4}(\phi)$ we find that $\alpha=1$; from $P_{6}^{6}(\phi)$ we find that $\alpha=1$; from $P_{7}^{7}(\phi)$ we find that $\alpha=0.977905 \ldots$, and so on. Thus, the even-even diagonal Padé approximants yield the value $\alpha=1$ while the odd-odd diagonal Padé approximants yield positive values for $\alpha$ that rapidly and monotonically approach 1 from below. 
In Figs. 4 and 5 we plot the real and imaginary parts of the effective potential at long scales that flows from massive potential $U_{0}(\phi)=\mu^{2} \phi^{2}-g \phi^{4}$. The Padé approximants $P_{4}^{4}(\phi)$ and $P_{7}^{7}(\phi)$ are plotted along the center line of the right Stokes sector. Note that for large $|\phi|$ the effective potentials that are obtained in the Padé approximation strongly resemble the plot of the potential in Fig. 3. The results for the massless case are equally impressive. These Figures provide strong support for the perturbative treatment proposed in this paper and give evidence that $\mathcal{P} T$-symmetric potentials give rise to valid and consistent quantum field theories.
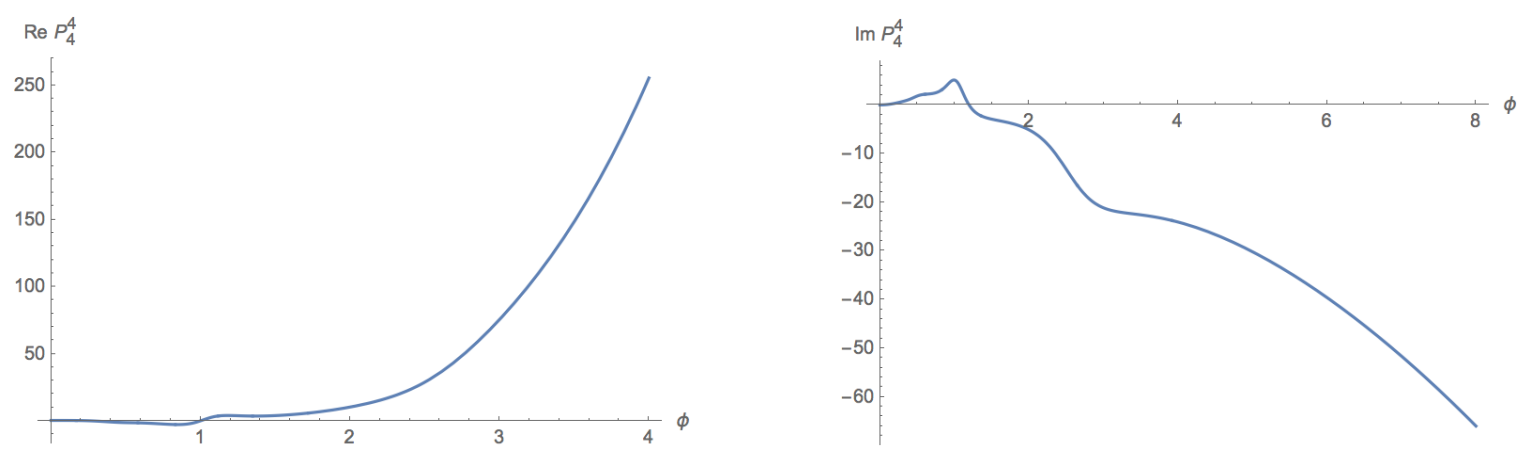

FIG. 4: Effective potential obtained from $P_{4}^{4}(\phi)$ plotted along the center line of the right Stokes sector for the negative-quartic massive theory $U_{0}(\phi)=\mu^{2} \phi^{2}-g \phi^{4}$ with $g=1$ and $\mu=1$. Note the good agreement with the plot of the potential in Fig. 3 .

\section{DISCUSSION}

The functional renormalization group in the potential approximation provides a seemingly simple nonlinear partial differential equation. However, we have shown using asymptotic analysis that the space dimension $D$ determines whether an initial-value problem well-posed or ill-posed; if $D<2$, the problem is well-posed and if $D>2$ the problem is ill-posed. For $\mathrm{D}=1$ we have used asymptotic analysis to investigate $\mathcal{P} T$-symmetric potentials. Under the Wetterich flow to the infrared we find that $\mathcal{P} T$ symmetry is preserved. Vacuum stability inherent in $\mathcal{P} T$ symmetric theories persists under renormalization. 

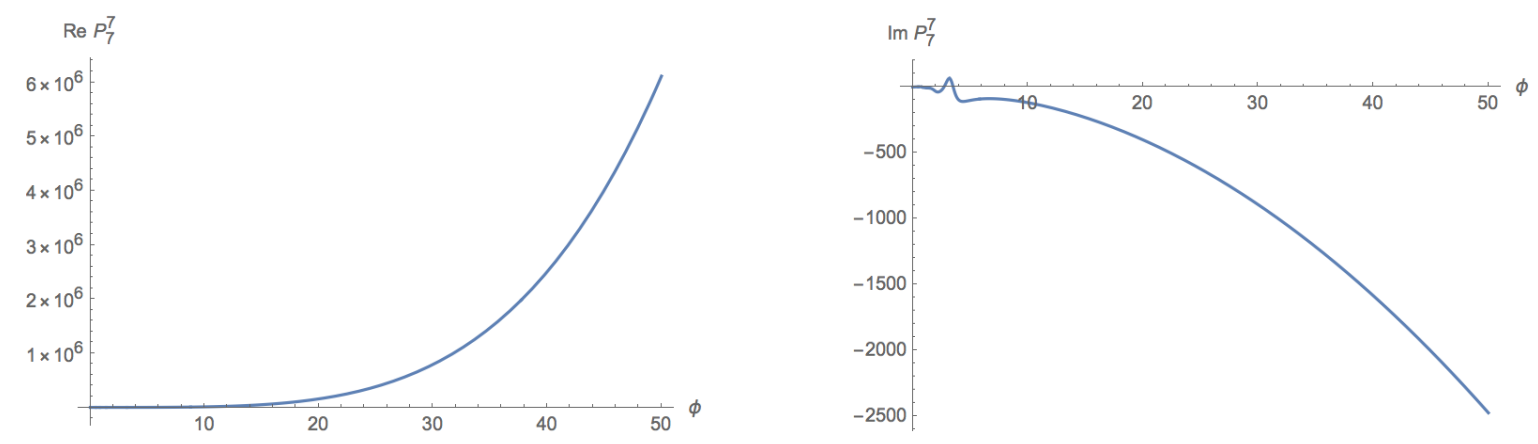

FIG. 5: Effective potential obtained from $P_{7}^{7}(\phi)$ plotted along the center line of the right Stokes sector for the negative-quartic massive theory with $g=1$ and $\mu=1$. Like the plot in Fig. 4, there is impressive agreement with the plot of the potential in Fig. 3 .

We have found that under the functional renormalization group transformation the effective action of $\mathcal{P} T$-symmetric theories flows to the infrared in a $\mathcal{P} T$-symmetric fashion. This indicates that our earlier discussions of $\mathcal{P} T$ symmetry and vacuum stability [16, 17] are relevant in a nonperturbative setting. The role of renormalization in selecting $\mathcal{P} T$-symmetric boundary conditions for field theories is an important question that requires further study.

\section{Acknowledgments}

We thank J. Alexandre and I. Saltas for helpful discussions.

[1] S. K. Ma, Modern Theory of Critical Phenomena (Benjamin, Reading, 1976).

[2] C. Domb and J. L. Lebowitz, eds. Phase Transitions and Critical Phenomena, Vol. 6 (Academic, London, 1976).

[3] H. Gies, Lect. Notes Phys. 852, 287 (2012).

[4] A. Wipf, Lect. Notes Phys. 864, 1 (2013).

[5] J. Alexandre, "BUSSTEPP 2017 lecture notes: Exact Wilsonian Renormalization," arXiv:1508.07763 [hep-th].

[6] C. Wetterich, Int. J. Mod. Phys. A 16, 1951 (2001). 
[7] J. Alexandre (private communication).

[8] C. M. Bender, Rept. Prog. Phys. 70, 947 (2007).

[9] P. E. Dorey, C. Dunning, and R. Tateo, J. Phys. A: Math. Theor. 40, R205 (2007).

[10] C. Domb and M. S. Green, eds. Phase Transitions and Critical Phenomena, Vol. 3 (Academic, London, 1974).

[11] D. F. Litim, Phys. Rev. D 64, 105007 (2001).

[12] J. A. Mueller and S. Siltanen, Linear and Nonlinear Inverse Problems with Practical Applications (SIAM, New York, 2012).

[13] C. M. Bender, S. F. Brandt, J.-H. Chen, and Q. Wang, Phys. Rev. D 71, 025014 (2005).

[14] C. M. Bender, M. Moshe, and S. Sarkar, J. Phys. A: Math. Theor. 46, 102002 (2013).

[15] C. M. Bender, D. H. Hook, N. E. Mavromatos, and S. Sarkar, Phys. Rev. Lett. 113, 231605 (2014).

[16] C. M. Bender, D. W. Hook, N. E. Mavromatos, and S. Sarkar, J. Phys. A: Math. Theor. 49, 45LT01 (2016).

[17] S. Sarkar, J. Phys.: Conf. Ser. 873012051 (2017).

[18] D. Lavis and G. M. Bell, Statistical Mechanics of Lattice Systems: Volume 2: Exact, Series, and Renormalization Group Methods (Springer, Heidelberg, 1999).

[19] G. A. Baker, Essentials of Padé Approximations (Academic, London, 1975).

[20] C. M. Bender and S. A. Orszag, Advanced Mathematical Methods for Scientists and Engineers (McGraw-Hill, New York, 1977). 